\title{
Symmetries as Humean Metalaws
}

\author{
Callum Duguid(D) \\ School of Philosophy, Religion and History of Science, University of Leeds, Leeds, UK \\ Email: calduguid@gmail.com
}

(Received 11 August 2020; revised 01 April 2021; accepted 22 July 2021; first published online 12 January 2022)

\begin{abstract}
Symmetry principles are a central part of contemporary physics, yet there has been surprisingly little metaphysical work done on them. This article develops the Wignerian treatment of symmetries as higher-order laws-metalaws-within a Humean framework of lawhood. Lange has raised two obstacles to Humean metalaws, and the article shows that the account has the resources available to respond to both. It is argued that this framework for Humean metalaws stands as an example of naturalistic metaphysics, able to bring Humeanism into contact with the practice of actual science without giving up on the central denial of necessary connections.
\end{abstract}

\section{Symmetry principles}

Humeanism is one of the main accounts of scientific law, offering a promising way to provide a metaphysics of lawhood without being committed to concepts like governance. Given that symmetry principles are central to the study of actual physical laws, Humeans ought to say something about their nature. However, this is a largely undeveloped area of the literature. This article develops a version of Humeanism that offers an account of symmetry principles and their relation to laws without having to introduce non-Humean necessary connections.

Symmetries of the laws are often granted prominence within the philosophy of physics. Philosophically, the claim that laws of nature hold universally is underwritten by the expectation that they are invariant under various spacetime symmetries, such as invariance under spatial translation. An example of a nonspacetime symmetry is permutation invariance, which is concerned with the statistical behavior of quantum particles and lies at the heart of the debate over their identity conditions (French and Krause 2006).

Within contemporary physics, symmetry principles are of central importance: "[I]t is only slightly overstating the case to say that physics is the study of symmetry" (Anderson 1972, 394). As is well-known from Noether's (first) theorem, there is a tight connection between symmetries and conservation laws: For every continuous global 
symmetry there exists a corresponding conservation law. To give just one example, conservation of energy is associated with invariance under temporal translations.

The status of symmetries within physics is not just theoretical, as they also play a heuristic role. The best-known example of this is the Eightfold Way classificatory scheme, developed in the 1960s. On the basis of symmetry considerations, GellMann and Ne'eman predicted the properties of a particle that would complete the baryon decuplet - the accuracy of this prediction was soon after experimentally verified. That the laws abide by symmetry principles is seen today as no mere accident: The latter are treated as guiding principles with which we expect future laws to accord (Post 1971). That the laws take on a certain form teaches us in turn about the sort of particle behavior that we might encounter, for example.

Yet despite all this, the role that symmetry principles have in an account of the metaphysics of laws is underexamined in the philosophical literature. ${ }^{1}$ That is an unfortunate state of affairs given the relationship between symmetries and the physical laws. The purpose of the metaphysics of science is to update our metaphysical views in light of contemporary science. This article is intended in exactly that spirit.

In the next section, I turn to one of the leading accounts of laws-the Humean regularity account-and explain how a natural extension of that account can incorporate symmetries. Symmetry principles are to be understood as metalaws: secondorder laws that describe patterns in the first-order laws. With the proposed view in place, I then consider two challenges that Marc Lange has raised for regularity accounts of this kind. The first, I argue, can be disarmed through closer examination of how possible worlds and counterfactuals interact on a Lewisian treatment. The second, concerning the naturalness of the properties in the metalaws, requires additional resources to answer. To respond, I draw upon the Better Best System Account as a way of avoiding commitment to redundant natural properties (Cohen and Callender 2009). The final section brings the metaphysics into contact with a challenge from the philosophy of physics. It has been claimed that symmetries are one area of contemporary science with which Humeans are poorly equipped to deal. This account of symmetries as metalaws functions as a response to that criticism, demonstrating that one can be committed to scientifically informed metaphysics without thereby having to give up a central element of the Humean worldview.

\section{Humean laws and metalaws}

Contemporary Humeanism is concerned with a denial of necessary connections between distinct existents. The instantiation of fundamental properties in this region of spacetime does not entail that any particular properties must be instantiated in that area of spacetime. Laws of nature, then, cannot constrain the goings-on of the world; they cannot ensure that events occurring here force certain events to occur there. Laws like that are typically described as governing laws, which ensure a necessary connection between what is here and what is there. ${ }^{2}$

For Humeans, the laws must instead be descriptive. There are patterns in the events that occur at our world and the laws summarize those patterns. To avoid

\footnotetext{
${ }^{1}$ With a few notable exceptions. See Lange (2009), Yudell (2013), and Hicks (2019).

${ }^{2}$ Although see Roberts (2008) for a proposed way to accommodate governance within Humeanism.
} 
well-known problems with the naive regularity account, in which any regularity-no matter how trivial - is taken to be a law, the standard Humean view is based on the Mill-Ramsey-Lewis account of lawhood: the Best System Account (BSA; Lewis 1983). According to the BSA, there are many different systems of descriptions of the world's events. Some of these systems are simpler than others, with fewer axioms or shorter chains of definitions. Some systems are stronger than others, in that they are more informative about the world's events. These virtues trade off against each other, as simpler systems tend to be less informative and vice versa. The system that achieves the best balance of these competing virtues is the best system. The laws are the universal generalizations that occur in the best system.

The question that arises for this account is how best to deal with the symmetry principles introduced in the previous section. If physicists draw a close connection between symmetries and laws, then the metaphysics of laws ought to make room for that connection. A recent proposal has been offered by Hicks (2019), who takes the symmetry principles to be further laws. Hicks imagines the Maximally Simple System (MSS), the output of a best systematization competition heavily weighted toward simple systems over strong ones, and argues that its content would be limited to symmetries. This shows us that the symmetries are "maxilaws": regularities that convey very general information about the world at a low cost in terms of simplicity. Hence, they are good candidates for inclusion in the best system.

This is ultimately based on Hicks's claim that the symmetries are primarily concerned with the events, much as the laws are. In support of this, he cites Wigner: "The geometric principles of invariance, though they give a structure to the laws of nature, are formulated in terms of the events themselves" (Wigner 1964b, 958). As we shall shortly see, however, Wigner is more commonly associated with a different interpretation of symmetries. A few paragraphs after the previous quotation, he goes on to say that: "It is good to emphasize at this point the fact that the laws of nature, that is, the correlations between events, are the entities to which the symmetry laws apply, not the events themselves" (Wigner 1964b, 959).

Setting aside the issue of motivation, there is a further difficulty facing the maxilaw view. Even if the symmetries are the content of the MSS, the Humean view aims to arrive at the same laws as contemporary physics, which are not limited to symmetries alone. Adding symmetries to a candidate systematization containing these laws will only come at a small cost to simplicity, but if the narrower information captured by individual laws is already included, it's not obvious how much additional benefit is provided by the maxilaws. If the laws are, say, invariant under spatial translation, then adding a proposition stating as much to a candidate system risks redundancy. ${ }^{3}$ This is particularly pressing given the interderivability of the spacetime symmetries and the conservation laws. Including both in a system would be redundant but leaving either category out would mark it as derivative in a peculiar way.

An alternative approach is to see symmetry principles as constraints: Because the range of, for example, particle behavior is limited by the symmetries that hold of them, only certain laws governing that behavior are possible. Historically, this view has its roots in the writings of Wigner, who describes a symmetry principle as "a superprinciple which is in a similar relation to the laws of nature as these are to

\footnotetext{
${ }^{3}$ This echoes a criticism of Humeanism that Lange makes $(2011,221)$.
} 
the events" (Wigner 1964a, 996). This is the metalaw view, where the symmetries are taken to be second-order laws that hold over the first-order laws. More recently, this has been developed in the work of Lange $(2007,2009)$. On his view, as the laws set constraints on the world's events, so too do the metalaws constrain the laws. Because Lange treats laws as possessing a greater degree of necessity than the events do, he treats metalaws as correspondingly more necessary than laws.

Phrased like that, it might look like the prospects for Humean metalaws are dim. After all, if metalaws are distinguished by their greater necessity and the Humean account is concerned with denying the existence of such necessity, then there is a clear tension in the position. However, there is a promising way out of this problem. ${ }^{4}$ The standard BSA accommodates the sense in which the laws are necessary by reducing the talk of necessity to that of possible worlds. Nomic necessity is defined in terms of those worlds that abide by our laws. An analogous move can be made for metalaws. Those worlds whose events and laws are in accordance with our symmetry principles are metanomically necessary. ${ }^{5}$

Let the laws be given by the BSA. The metalaws will be the universal generalizations present in the best systematization of the first-order best system. The results of the BSA can be summarized in various ways, some simpler than others and some more informative. Simply listing all of the facts concerning the laws will be maximally informative, in the same way that a list of all the facts concerning the world would be maximally informative in the BSA. As we get a trade-off of theoretical virtues against each other when systematizing the results of the BSA, a competition can be run to find which second-order system achieves the best balance. Look at the universal generalizations present in the higher-order system: These are the metalaws. ${ }^{6}$

One might wonder why we should not just take all the regularities that hold of the laws to be metalaws. After all, because the laws are (trivially) nomically necessary, we don't need to worry about any pattern present amongst them being accidental. Well perhaps not, but we still need a distinction between patterns like spatial invariance on the one hand and "by-product" patterns on the other. ${ }^{7}$ Suppose that, expressed mathematically, none of our laws contains a term raised to the ninety-seventh power. This would constitute a pattern over the laws, albeit a rather uninteresting one. But there's no reason to take this to be a second-order law. It instead appears merely

\footnotetext{
${ }^{4}$ This is suggested briefly in Lange (2007, 479): "Would the meta-laws be the members of the best system of truths about the first-order laws (i.e., about the best system of truths about the Humean mosaic)?" This article expands on what a positive answer to Lange's question entails. Note that Lange rejects the view based on the obstacles he raises in Lange (2011).

${ }^{5}$ As "meta-physical possibility" is ambiguous here, it will be clearer to stick to nomic and metanomic as the appropriate terms.

${ }^{6}$ An anonymous referee wonders whether there might be metalaws that are not universal generalizations. One example offered in Lange $(2009,186-88)$ is determinism, understood as a completeness requirement on the laws: There are enough nonstatistical first-order laws to determine the universe's states at all moments from its state at any one moment. I suggest that the Humean described here allow for such claims to enter the second-order best system as simple and informative facts about the laws but deny them the label of metalaws. This is analogous to how Lewis $(1983,367)$ treats important first-order facts like initial conditions. While the (in)determinism of the laws is certainly a matter of interest to science, I do not take treating them as metalaws specifically to be a nonnegotiable aspect of scientific practice.

${ }^{7}$ The introduction of "by-product" to this terminology is due to Lange (2007).
} 
coincidental, connected to no deep fact about the character of the laws. Requiring the metalaws to be generated by a second round of systematization competition prevents us from according such by-products with any importance, in the same way that the BSA blocks any old regularity from assuming the mantle of lawhood.

This second-order BSA is not a replacement for the original. It had better not be because it relies on the output of the first-order competition for the facts it systematizes. In principle, one could continue this extension process by examining a thirdorder BSA and so forth. Note, of course, that the mere fact that the framework for a third-order account is available does not guarantee that there will be any associated metametalaws. Should the world be sufficiently disordered it will lack the sort of striking regularities that the first-order BSA will describe as laws. Similar comments apply to the second-order BSA: Should the first-order laws fail to exhibit sufficiently important regularities, there will be no metalaws. This will obviously continue to hold for the higher-order best systems, so there is no guarantee that by moving to a thirdorder BSA we will find metametalaws.

As the BSA is intended to treat the virtues involved in theory-building as constitutive of lawhood, our best guide to how high the (meta)law structure rises is the practice of actual science. One of the motivations for caring about the output of the BSA is that we need an account of laws that allows them to play the roles scientists use them for, such as prediction and explanation. Similarly, the central importance of symmetries in physics provides ample motivation for constructing a second-order BSA. Whether or not we should care about the output of a third-order BSA depends on whether regularities in the metalaws are being appealed to by scientists in, for example, the resolution of appropriate counterfactuals. That is an empirical question, and one that is orthogonal to the unhelpful issue of whether metametalaws "really exist."

That concludes our overview of the basic Humean claim concerning metalaws. There are important questions to be answered concerning how well such Humean metalaws play roles analogous to the laws in, say, explanations and inferences. I set such questions aside because they are the focus of other work on the subject (see Yudell 2013). I follow the literature in also setting aside challenges to Humean metalaws that are analogies of those faced by Humean laws, such as the concern that coincidental regularities might be strong and simple enough to buy their way into the best system (see Lange 2011, 217). The article instead draws out further details of this Humean account by examining how it deals with two challenges that Lange has raised against it. The first of these, that Humean metalaws fail to be sufficiently robust in ordinary counterfactual reasoning begins the next section.

\section{The first challenge}

In addition to suggesting the natural way in which to extend Humeanism to cover metalaws, Lange also offers two obstacles for Humeans to overcome. The first is concerned with the relationship between metalaws and counterfactuals. In the standard Lewisian treatment of counterfactuals, laws play an important role. They are especially resilient under counterfactuals in a way in which matters of particular fact are not. This is reflected in Lewis's (1979) list of priorities for counterfactual evaluation: Avoiding widespread law violations is of the highest importance. In ordinary contexts, the most similar possible worlds are those that contain no miracles other than those required to bring about the counterfactual antecedent. Similarly, when 
assessing counternomics the closest worlds are those that minimize the number of required metalaw violations. If we want to know what planetary orbits would be like in a world where the gravitational force was an inverse cube law, we need to assume that spatial invariance holds at that world. This only holds true in ordinary contexts, of course. There is nothing preventing us from asking what would happen in a world with both law and metalaw violations. But in ordinary contexts metalaws show a resilience similar to that shown by laws. Because treating the metalaws in this way is a feature of standard scientific practice, any metaphysical account of them must be able to capture their resilience.

Laws are resilient under antecedents concerned with differences of particular fact and metalaws are resilient under antecedents concerned with differences of laws. The problem Lange sees is that the metalaws offered by the Humean account may not be resilient under the former kind of antecedents, unlike the metalaws found in scientific practice. In other words, ordinary counterfactuals like "Had I struck the match at spatiotemporal location L, it would have caught fire" cause problems for this Humean treatment of metalaws.

To assess this counterfactual, we consider worlds at which my counterpart does strike the match at location $L$. Those closest to ours will have similar laws to ours. If determinism holds, they will not be quite the same because, from the perspective of our laws, a small miracle is required for the match to be struck. What then are the laws of the closest world? At minimum, they must be exceptionless regularities. One suggestion is that they are the laws of our world, but with the addition of a clause like "except at spatiotemporal location $L$, where the following occurs." ${ }^{8}$ This allows for the "miraculous" striking of the match to occur without any of that world's laws being violated. But while that world's laws have not been violated, that world cannot have the same metalaws as our own. If the laws pick out some spatiotemporal locations as special, then they cannot be invariant across spacetime. This conclusion is at odds with the use of metalaws within science, which takes them to be more resilient than laws.

While Lange takes this to be an issue with the Humean account, he is not insensitive to the point that there are other ways to distinguish events. This gives rise to the alternative route that he offers. Let $C$ refer to some combination of natural properties that is present at the striking of the match. Now replace the reference to spatiotemporal location $L$ in the clause with a reference to combination $C$. If this were added to our laws, we get a candidate system of laws for this close world. The laws of that world would not be violated for the same reason as before. Better still, the metalaws of that world would not need to differ from our own as no spatiotemporal location is treated differently from any other, and we have no reason to think that our metalaws make reference to invariance across different natural properties.

After offering this route to Humeans, Lange rejects it out of concern that combination $C$ might be multiply instantiated. Should a possible world have laws that include a clause mentioning $C$, then what we would regard as a miracle can occur whenever that particular combination of properties is instantiated. This will happen at the striking of the match, of course. But properties can be instantiated at multiple different times and places. Given the Humean rejection of necessary connections,

\footnotetext{
${ }^{8}$ This suggestion, offered by Lange (2011), echoes comments made in postscripts to Lewis (1979), printed in Lewis (1986a, 54-55).
} 
there is nothing to prevent combination $C$ from being instantiated more than once at the world. Preventing this requires some form of necessary connection that blocks $C$ from being repeated, which is clearly not an option for Humeans here. Should $c$ be instantiated more than once at the world, there would (from our perspective) be multiple miracles at the world. ${ }^{9}$ Yet Lewis's own priorities for closeness of worlds require that miracles be minimized. In short, if the clause mentions only natural properties then there is nothing to guarantee that a world whose laws have that clause will not have multiple miracles and so be unsuitable for evaluating counterfactuals.

For Humeans who accept Lewis's treatment of counterfactuals and possible worlds, however, the objection misses its target. Lange is right to point out that nothing prevents $C$ from being instantiated repeatedly at a world. It is also true that a world with multiple $C$-instantiations could have the same laws as a world with only a single $C$ instantiation. (There are limits though: A system of laws that recognizes too many special cases is unlikely to be counted as the best system.) Yet neither of those facts create problems for evaluating closeness of worlds. It is not as if evaluation of counterfactuals requires that we first decide on the laws that must occur at the closest world and then take any world with those laws and treat it as the closest regardless of the events that occur at the world. If it were, then Lange's objection would have some force because merely deciding on the laws is not sufficient to pick out worlds with only a single miracle. But this is a mistaken picture of Lewis's account.

Worlds differ from one another in that they have different patterns of instantiation of natural properties. Combination $C$ will be instantiated once at some worlds, multiple times at others, and not at all at still more. ${ }^{10}$ Some of the worlds where $C$ is instantiated a single time will have laws like our own, only with the addition of clauses as suggested. Some worlds where $C$ is instantiated multiple times will have exactly the same laws as single- $C$ worlds. Some worlds where $C$ is instantiated will have wildly different laws or perhaps none at all. These worlds are not treated equally when it comes to counterfactuals. When assessing how close these worlds are to our own, we turn to Lewis's list of priorities. One of the priorities given is to minimize the number of miracles that occur. This minimization is not meant in some creative sense where we influence other worlds, but simply that those worlds which happen to have more miracles are less similar to our own than worlds with fewer miracles. This is why multiple instantiations of $C$ is not an issue. Nothing prevents there from being worlds with multiple instantiations, but they are less similar to our world than those with only a single instantiation of $C$. So when assessing counterfactuals like "Had I struck the match it would have lit" we only look to worlds where the miraculous lighting of the match happens a single time.

Evaluators of counterfactuals do not typically attempt to figure out the precise details of combination $C$ that would occur at minor miracles like the lighting of

\footnotetext{
${ }^{9}$ Lange $(2011,219)$ writes: "However, there would then be nothing to prevent this combination $C$ of properties from being instantiated not only at the striking of the match, but once again somewhere somewhen. In that event, for the law to hold in the [relevant possible world], there would then have to be another departure from the actual laws - another small miracle."

${ }^{10}$ The principle of recombination-roughly that anything can exist or fail to coexist with anything else-assures us of this by filling out the space of possible worlds (see Lewis 1986b, 87-92). In particular, it ensures that there will be worlds that match the actual world up until the relevant time, then instantiate $C$ once to provide the requisite miracle, and then never again instantiate $C$.
} 
the match. As an anonymous referee suggests, one might wonder whether this constitutes a problem for the Humean account: Does it misrepresent what ordinary counterfactual reasoners do in practice? It does not. On Lewis's account, counterfactual evaluators are concerned with the happenings at those worlds that minimize miraculous violations of the actual laws. They are not usually concerned with what the laws of those similar worlds are ${ }^{11}$ Consequently, they are not usually concerned with specifying appropriate clauses to ensure that the laws of the similar worlds are exceptionless.

\section{The second challenge}

To understand Lange's second objection (2011, 220-21), we must first note that the canonical BSA requires every system to be stated in a language whose predicates correspond to perfectly natural properties. The usual reason given for this is that without this restriction the account will misidentify the laws (see Lewis 1983, 367-68). Consider the predicate $F$ that holds of all and only those things in our world. Then the system consisting of the single sentence $\forall x F x$ will get counted as the best. It is simple, almost certainly simpler than its competitors. It is also maximally strong, for such a system will hold at only those worlds indiscernible from our own (should there be any). Yet despite being the best system, it does not give us the laws. For this sentence will imply all the truths of our world and hence all the regularities. Consequently, every one of the world's regularities will get counted as a law: an absurd conclusion! Not only would this obliterate the distinction between laws and accidental regularities that the BSA was supposed to maintain, it would make a mockery out of scientific practice. We would not discover the best system through empirical investigation but through simple armchair reasoning.

Clearly something must be done to prevent this degenerate system from being counted as best. The specific language requirement does exactly that as the predicate $F$ does not correspond to a perfectly natural property. To use only those predicates referring to perfectly natural properties in stating the system consisting of $\forall x F x$ as its one axiom would require a very long complicated chain of definitions from those basic predicates to the predicate $F$. Such a chain would cause the system to score very poorly on simplicity and hence no longer be counted as the best.

But while this reason for stating all systems in Lewis's favored language is wellknown, there is another reason. ${ }^{12}$ Simplicity is a language-dependent notion, defined relative to a system of basic predicates. A system will be simpler in one language than in another. For example, a system containing the sentence "All emeralds are green" will get counted as simpler than "All emeralds are grue" in a language whose basic predicates include "green" but not "grue" (Loewer 1996). Exactly the opposite result occurs if we consider a language whose basic predicates include "grue." Because simplicity is relative to language, simplicity comparisons of systems stated in different languages are impossible.

\footnotetext{
${ }^{11}$ This is why the four priorities for similarity of worlds that Lewis offers in his (1979) make no mention of the laws at nonactual worlds. See also his discussion of miracles on pp. 468-69, which dismisses the relevance of such laws.

${ }^{12}$ Cohen and Callander (2009) reminded metaphysicians of this, but we should also credit Lewis (1983) who brought up exactly this point as well.
} 
One option when faced with this problem is to follow Lewis's example and demand that all systems be stated in the same language: one whose predicates refer to perfectly natural properties. It is the business of physics to discover what the perfectly natural properties are. As no one thinks that our current physics has achieved a final theory, it is reasonable to assume that a complete inventory of the perfectly natural properties has not yet been completed. But if we are optimistic then we might believe that we understand some of what the fundamental properties are. This is a suitable language because it respects the distinctions that are present in the world's fundamental character. To reuse a well-worn metaphor, if we were to carve the beast of reality at the joints, we would be carving it along lines demarcated by such properties.

Suppose - for now - that this is the best solution to the issue of selecting a language. The best system is the system that has the best balance of theoretical virtues when stated in the language whose predicates refer to perfectly natural properties. It is natural, then, to extend this to Humean metalaws. The second-order best system must also be stated in a particular language to avoid a similarly degenerate choice of predicates: If $G$ is the property of being in a world where (the sentences in the firstorder best system) are exactly the sentences in the first-order best system, then $\forall x G x$ will be the second-order best system. This would be a disaster, for such a system would entail all the first-order laws and so all those laws would also get counted as metalaws. This parallels the problem in the first-order case exactly: It gets the wrong results and arrives at them through a priori reasoning instead of empirical investigation. We might hope that by adopting the same language that we did in the first case we might avoid the problem in the same way.

Unfortunately, that move is not as promising here. It would block predicates like $G$, given that it is no more natural than $F$ was. But, as Lange points out, it blocks rather too much. Metalaws are not stated in terms of perfectly natural properties, but rather ones like covariance under temporal displacement:

Being time-displacement covariant is a mathematico-logical property, and it is a property of regularities, not of events. Any property that involves being somehow related to the best system for describing the Humean mosaic is not a natural property: if we described the mosaic in terms of the complete global pattern of instantiation of natural properties, then it would be redundant for us to add a description of the best system for describing the mosaic, whereas descriptions in terms of natural properties are not redundant, Lewis says. (Lange 2011, 221)

The relationship between Lewisian natural properties and mathematics has not been heavily developed in the literature, although it has been claimed that mathematical practice gives us reason to accept that there are natural mathematical properties. ${ }^{13}$ Instead, I take the underlying reason why the properties referred to by the secondorder best system cannot be perfectly natural is their redundant nature. The comment that we are dealing with properties of regularities is a comment on that redundancy. Suppose that we have a description of the fundamental nature of the world in terms of perfectly natural properties and relations. A further description of those relations would not add any new information: Anything featuring in it would

\footnotetext{
${ }^{13}$ For example, Tappenden $(2008,258)$ and Lange $(2016,338)$.
} 
either be contained within the original description or would be entailed by it. However much creatures like us would find the second description useful, it is redundant. ${ }^{14}$

For this reason, the second-order best system will not be stated in terms of perfectly natural properties. This is a problem if we require all systems to be stated in a language that takes these to be its basic kinds. Because metalaw properties are not perfectly natural, a translation of them into perfectly natural ones would be, at best, a blow to any candidate system's simplicity. Less optimistically, the gruesomeness of the resulting sentences might be enough to destroy the chances a system had of being the best.

A Humean could push back against this conclusion by reminding us that we are dealing with the Best System Account, not the Great System Account or even the Fairly Good System Account. For a system to count as best it need only be better than its competitors - it does not need to score particularly highly on whichever metric is used to assess the balance of strength and simplicity. All we've seen is that a system consisting of what we currently take to be metalaws will not be particularly simple when stated in terms of perfectly natural properties. It has not been shown that rival systems will fare any better. Going further, if no candidate system is able to score highly then it may not make much sense to talk of the scores being low. On this view, it is entirely irrelevant that second-order systems of regularities will not be as simple as we might have initially thought.

Unfortunately, this is not an ideal response. While Lange has not demonstrated that rivals to the system that we think gives us the metalaws will achieve a better balance, this reply has also not supplied a reason to think that they will not. It is a weakness of the response that it simply leaves the matter open. Note that this is stronger than the old concern that the laws of the first-order best system may not match the scientific laws (van Fraassen 1989, 53). The problem arises in that context because the predicates that scientists use might fail to correspond to the perfectly natural properties. In this context the issue isn't that they might fail to correspond, but that we have reason to think that they won't: The relevant properties are just not candidates for perfect naturalness on account of their redundancy.

Fortunately, there is a model for how we might answer the question of language: the Better Best System Account (BBSA). ${ }^{15}$ Presentations of the BBSA are concerned with offering a competitor to, or development of, the orthodox BSA. As such, they make no mention of symmetries or metalaws. There is no reason, however, why we could not modify the account to include these in the same way that the BSA can be extended. The focus of the remainder of this section is to describe how the BBSA can be extended to cover metalaws and explain why this approach will not run afoul of Lange's concern regarding language.

The core insight behind the BBSA is that the original BSA needs adjustments to properly accommodate laws of the special sciences and that modifying the vocabulary used is the first step to making these adjustments. Notice that the language problem identified in the preceding text applies not only to metalaws but also to any system of

\footnotetext{
${ }^{14}$ A similar point is made by Wigner (1964b, 957-58).

${ }^{15}$ For examples of this move, see Roberts (1999), Halpin (2003), Schrenk (2008), Schrenk (2014), Cohen and Callender (2009), and Callender and Cohen (2010).
} 
laws not stated in terms of perfectly natural properties. Because the perfectly natural properties are taken to be discovered by physics and are without redundancy, there is little hope that any regularity of the special sciences will be present in the best system when stated in the relevant language. There is some controversy as to whether there are any laws to be found in the special sciences. ${ }^{16}$ But this is a question that should be resolved by examining the appropriate field and not one that is settled by metaphysicians alone. Our metaphysics of laws should at least allow for the possibility of laws in domains other than fundamental physics, and the BBSA is one way to achieve this goal.

The central change to the BSA that Cohen and Callender make is to remove the claim that any particular choice of basic predicates is picked out by the world as objectively special. There is no sense in which any language that we choose to formulate systems of laws in is better than any other - at least, that is, no sense that does not make appeal to our particular contingent interests or abilities. As an example of this, we might compare a language with green and blue to one with bleen and grue. The former language is easier for us to work with, but this does not mean that there is anything objectively better about it when it comes to picking a language in which to formulate laws.

According to the BBSA, the laws are still given by the system that best balances the competing virtues of strength and simplicity. However, rather than having to be stated in a single specific language, systems of laws can be formulated in any language. Lawhood, then, becomes a language-relative notion. We might still take which regularities are the laws to be an objective matter; we do not get to freely choose what the laws are. But this is a constrained notion of objectivity because they are objective only relative to a choice of language. Each language has its own competition for best system, and different languages might have different laws. Some languages might not even recognize any laws, should they lack the resources to describe the world's regularities. Relative to the language used by physicists, the laws will be those of physics. Relative to the language used by economists, the laws will be those of economics (assuming that there are such laws).

At this point, the extension of the BBSA should be clear. Begin with a framework in which laws are language-relative and then extend this to cover higher-order regularities. As the competition for best system can be run in any language that we choose, so too can we then run the competition for the best second-order systematization of that system in the same language. Whether that competition will offer us metalaws depends on two things. First, it depends on what the first-order system is like. Should this be sufficiently disordered or lacking in content, we might not get a strong and simple system of second-order regularities describing it. That's the right result: Whether there are metalaws ought to depend on what the laws are (which in turn depends on the way the world is).

Second, whether we get metalaws depends on the language chosen in the way noted by Lange. We should not take this to be a troubling concern. After all, the motivation behind the BBSA is that we need to look to the practices of the relevant scientists to know what language is appropriate for assessing the associated best system. Let us do exactly that: Pay attention to the terminology used by scientists

\footnotetext{
${ }^{16}$ For example, Beatty (1995) argues that there are no distinctively biological laws.
} 
working in the field under consideration and consider a language that includes such predicates. Return to covariance under temporal displacement as an example term. If physicists are wont to work in a language that includes this term then we should assess the best systematization of first-order physical laws in a language that includes it. ${ }^{17}$

While metalawhood is not tied in a metaphysical sense to any specific choice of language, such fixing of a language is required for practical purposes: Failure to do so would lead to individuals talking past one another when discussing metalaws. This article's position is that our usage of the language of physics is an implicit recognition of that need. While there are systems of laws and metalaws available for different choices of language, these alternatives are set aside when we speak of "the metalaws of physics." There is no need for naturalistic Humeans to prescriptively claim that physicists must use the language they do to arrive at the metalaws we recognize. ${ }^{18}$ Scientists are free to work in whichever language they find most productive in advancing their field. Not everything is up for grabs: The mosaic of events ultimately determines what regularities are available to be described. But certain languages, and their associated ways of carving up the world, will make it easier to find strong and simple regularities. Similarly, a choice of language will be better to the extent that it helps physicists discover such heuristically useful principles as metalaws.

On this view, there is no requirement that physicists use a language that includes predicates like covariance under temporal displacement and so judges the spacetime symmetries to be simple. But the importance of the metalaws to contemporary physics is motivation enough for physicists to do so. Progress in physics would be inhibited by working in a language without such predicates and so a choice of language that delivers the known metalaws is better to that extent. The fact that it fails to meet the standards of perfect naturalness is of no concern here because we have dropped the requirement that all predicates in the competition language need to correspond to such properties. ${ }^{19}$ The second problem that Lange raises for extending the BSA simply does not occur when we are instead extending the BBSA.

\footnotetext{
${ }^{17}$ There is a tension here between using physics as a guide to perfectly natural properties and the desire to let "the language of physics" to be set by what terms actual physicists use. I suggest that suitably naturalistic Humeans should favor the latter consideration and bite a bullet here: There will not be a one-to-one correspondence between the predicates used in physics and the perfectly natural properties.

${ }^{18}$ Earlier I considered the reply that the best system need only be better than its competitors and found it wanting because it could not guarantee that we'd arrive at the metalaws. As an anonymous referee asks, why not be concerned here too? The reason is that extending the BBSA drops the sense in which the second-order best system is unique, as there will be competition for best in every language. With a unique best system, it matters whether it contains the metalaws: Failing to do so means failing to reflect the practice of physics. Without uniqueness, we can be confident that there will be a language available where the second-order best system contains the metalaws. I take the claim of the BBSA to be that physicists have shown that they prefer to work in such a language.

${ }^{19}$ This separates laws from natural properties, although Humeans do not have to drop the latter entirely: They can earn their keep in other areas of metaphysics. See Dorr and Hawthorne (2013) and Dorr (2019) for the other roles natural properties play.
} 


\section{Putting the framework to use}

The importance of offering a Humean account of symmetries goes beyond capturing an aspect of scientific practice: It provides a way of disarming a critique from the philosophy of physics concerning necessary connections and categoricity. McKenzie has argued that the Humean package of categorical fundamental properties and contingent laws of natural is untenable when one examines how these are connected to the symmetries of quantum theory. ${ }^{20}$ In this section, I will both explain the alleged tension and show how the metalaw interpretation accommodates this talk of symmetries without commitment to irreducible necessity. Lange (2007) challenged philosophers of natural law to offer an account of metalaws. This section shows that an answer to that challenge can do further work illuminating how Humeans should understand (meta)nomic necessitation.

To begin, assume that the laws of quantum theory are fundamental. The fundamental entities are the particles described by the Standard Model, which come in multiplets: families of particles that possess differing magnitudes of the same determinable properties. These multiplets are described through the mathematics of group theory, a consequence of which is that each family's interactions will be associated with certain symmetries. Now consider a possible world that contains duplicates of some actual particles described by the Standard Model:

[I]f we understand the laws operative there along quantum-mechanical lines it follows that those laws must possess the symmetry of the laws of the actual world. But that represents a hugely informative and non-trivial constraint on the laws that any such set of duplicates can accord with. (McKenzie 2014, 55; emphasis in original)

Humeanism - particularly in the style of Lewis - is built upon a foundation of categorical properties, those that do not impose any constraints upon the laws in which they enter. For example, there are worlds where the electrostatic force described by Coulomb's law declines as the inverse cube of distance, rather than the inverse square. Whether there are any limitations on the form that a law for charged particles can take remains an open question; McKenzie points out that various metaphysicians seem to believe that there are no nontrivial limitations whatsoever. This ties in with the contingent status of laws: Because there are worlds with duplicates of charged particles that do not obey Coulomb's law, that law cannot be metaphysically necessary. If there are nontrivial constraints on the behavior of particle duplicates, then their kind properties are not categorical. If those properties are not categorical, then there are restrictions on the laws that can describe their behavior and so the laws are not contingent. Hence necessity of the actual symmetries threatens the Humean package of commitments.

Despite that, McKenzie does suggest that a reconceptualized Humeanism might still be viable. It is worth pausing to explain why this new look is an uncomfortable fit. The core of her suggestion is that the relation between group theory and multiplets is a mathematical one:

${ }^{20}$ McKenzie's (2014) point is that the entire canonical debate needs to be rethought, though defending the anti-Humean position in that debate is beyond the scope of this article. 
[I]s it not at bottom a mathematical fact that a set of particles, defined by a given set of determinate values, cannot participate in laws of quantum-theoretic form with arbitrary symmetry structure? (McKenzie 2014, 59; emphasis in original)

This emphasis matters because contemporary Humeans are not normally opposed to facts holding of mathematical or logical necessity. So, McKenzie continues, if mathematical necessity is not a problem and the determination of the laws for a set of particles is born of mathematical necessity, then there is nothing in this picture that Humeans need to be alarmed about. They should instead abandon the commitment to contingent laws as based on outdated physics and embrace a new world of necessary fundamental laws.

My concern is with taking the introduction of necessity as acceptable whenever the particles in question are defined by the mathematical values following from the relevant symmetry. No part of that maneuver is specific to the theory being a quantum one. The same move works in the classical context too: As long as we define the entities in question by the mathematical values that some theory assigns to them, we can then claim that there are unmysterious necessary connections between them of a mathematical nature. But if we can get necessity in the world whenever properties can be represented mathematically, then the floodgates have truly been opened. Humeans have been unwilling to treat necessitation in mathematical representations as entailing necessitation in nature, despite the fact that properties have been defined in terms of mathematical values long before quantum theory.

A more promising line for Humeans to take in response to McKenzie's problem is to utilize this article's approach to symmetries as metalaws. The difficulty arose because of the claim that duplicates of actual particles must behave in ways that are ultimately captured by appropriate symmetry principles. On the face of it, that is a similar claim to the usual ones involving laws. Two like-charged particles, for example, must accelerate away from one another in the absence of any other forces. Because the laws are necessary, duplicates of those particles in similar conditions must also accelerate away from one another. But for all this talk of the connection between laws and necessity, Humeans have a standard line of response about why particles always behave in ways described by the appropriate law. This is not because the law makes them do so, it is because the law is an accurate description of their behavior. That we expect duplicates to behave similarly is understood as a restriction of possible worlds: Among those worlds where our physical laws are true, particles behave in the same way.

As for laws, so for metalaws. If we understand the relevant symmetries using this article's framework, they end up as higher-order descriptions of the mosaic, which includes the particles and their behavior. That actual particles behave in ways described by the symmetries is because those symmetries are accurate descriptions of what happens (albeit indirectly; the metalaws are more directly concerned with the laws). As before, the related necessity is reduced to restricted quantification over worlds. The symmetries hold of metanomic necessity, which is to say that there is a sphere of possible worlds whose events occur in accordance with the actual metalaws.

Counterfactual suppositions about the behavior of particle duplicates will often have them behaving in ways described by our symmetries. That's not because these 
are the only possible worlds but because the resolution of counterfactuals standardly depends on contextual factors that restrict the range of worlds under consideration. In the same way that we typically try to avoid widespread violations of laws when assessing counterfactuals, we attempt to minimize metalaw violations. As such, worlds that our symmetries are true of will often be the closest in this assessment. In other contexts, such as the resolution of counterlegals concerning classical worlds, the symmetries of the Standard Model will not be relevant. At those worlds, counterparts of actual particles behave in ways that our actual physics does not describe.

What of the claim that our understanding of, say, charge is tied to group theory and hence to particular symmetries? Then particles at worlds described by a different symmetry structure cannot truly be said to have the property of charge. Is that not a restriction of categoricity and the introduction of necessity?

Tying the identity of a property to its nomological role would introduce an antiHumean form of necessity. Such nomic necessitarianism ${ }^{21}$ is committed to the actual properties only being associated with the actual laws - alien laws must cover alien properties. However, this position is not uncontroversial. In the present debate, it cannot merely be asserted but must be argued for. In the remainder of this section, I turn to two arguments that McKenzie deploys in defence of necessitarianism.

The first argument is that if we are discussing duplicates of entities, we first need to settle what those entities are. If we agree that they are quantum entities, then they are associated with certain symmetries and we must use laws of a quantum template to describe them. Kind duplicates of quantum entities are also quantum entities and so must fit the same nomological structure. Hence, they cannot behave in arbitrary ways.

What is it for an entity to be a quantum entity? The most obvious response is that it is the sort of entity described in a quantum theoretic way. But if this implies that duplicates of that entity are also quantum entities and so must be described in a quantum theoretic way too, then it straightforwardly begs the question. If calling an entity quantum at the outset enforces a limitation of its otherworldly behavior, then of course we must use quantum theory to describe it. But that necessity was introduced by calling the entity a quantum one. We cannot demonstrate that duplicates of elementary particle kinds are modally tied to the symmetries of the Standard Model by first limiting the possibilities we are willing to consider to those where the relevant laws possess the same regularities as our own. Restricting our attention to the sphere of metanomic possibility will inevitably result in the conclusion that duplicates will act in accordance with the actual symmetries. However, if an entity's being quantum does not impose any such limitation, then it is difficult to see what point there is in attaching the label.

The necessitarianism that McKenzie advances might be underwritten by a particularly strong form of naturalism. While it is common for philosophers to treat science as giving us reason to think that certain arrangements of events are possible, one might go further and suggest that science is the only guide to possibility. The only possible worlds that we should take seriously are the physically possible ones. ${ }^{22}$ This

\footnotetext{
${ }^{21}$ In the terminology of Schaffer (2005).

${ }^{22}$ In Schaffer's (2005) terminology, this would be modal necessitarianism, which recognizes only a proper subset of the worlds recognized by nomic necessitarianism.
} 
has the advantage of providing an explanation for why a particular arrangement is a genuine possibility that goes beyond conceivability or metaphysical fancy.

It does, however, also come with a substantial downside. One important use of possible worlds is providing truth values for counterfactuals. By replacing metaphysical possibility with physical possibility, this strong naturalism would fail to appropriately back the truth values of a wide range of counterfactual claims. If there is no world in which the antecedent of a counterfactual conditional is true, then the entire conditional ends up being trivially true. This might not be a great cost when it comes to fantastical scenarios involving sorcerers and talking donkeys! But, as McKenzie emphasises, the actual world is a quantum one. If the actual laws rule out classical worlds then any counterfactual concerning classical scenarios is automatically true. Given that scientists make appeal to such counterfactuals, that is a large bullet to bite.

Such considerations are not decisive, of course. Hard-core naturalism might be made compatible with nontrivial counterfactuals by adopting a hybrid view of possible worlds, where some worlds really exist and others are just useful fictions. Evaluation of such a proposal is a significant project and does nothing to lessen the upshot of this section. Even a strongly naturalistic philosopher cannot move straight to the conclusion that particle duplicates act only in ways described by the actual laws and symmetries. There are costs to that claim regarding how best to deal with various counterfactuals, and these costs can be weighed up against the costs of accepting a full plurality of worlds.

The aim of this article is to further develop the Humean account of metalaws, which is compatible with that account coming at a cost in its commitments. Acceptance of the language-relativity of the BBSA might be another of those costs, as suggested in the previous section. While a specific commitment regarding the language of candidate systematizations is not necessary to respond to McKenzie, it is required to overcome Lange's second objection. This section demonstrates the value of developing such a Humean account of metalaws: Responding to Lange's challenge provides Humeans with a way to interpret physical symmetries without thereby being having to accept irreducible necessity. ${ }^{23} \mathrm{I}$ leave it to the reader to decide which costs they are willing to pay for such an advantage.

Acknowledgments. I would like to thank Steven French, Juha Saatsi, Rob Knowles, and Víctor Durà-Vilà for their extensive discussion of this material. Thanks also to three referees for their helpful comments on earlier drafts.

\section{References}

Anderson, Paul W. 1972. "More Is Different." Science 177 (4047):393-96.

Beatty, John. 1995. "The Evolutionary Contingency Thesis." In Concepts, Theories, and Rationality in the Biological Sciences, edited by Gereon Wolters and James G. Lennox, 45-81. Pittsburgh: University of Pittsburgh Press.

Callender, Craig, and Jonathan Cohen. 2010. "Special Sciences, Conspiracy and the Better Best System Account of Lawhood." Erkenntnis 73 (3):427-47.

Cohen, Jonathan, and Craig Callender. 2009. "A Better Best System Account of Lawhood." Philosophical Studies 145 (1):1-34.

Dorr, Cian. 2019. "Natural Properties." In The Stanford Encyclopedia of Philosophy, Fall 2019 ed., edited by Edward N. Zalta. https://plato.stanford.edu/entries/natural-properties/

\footnotetext{
${ }^{23}$ Thanks to an anonymous referee for pushing me for clarity on this.
} 
Dorr, Cian, and John Hawthorne. 2013. "Naturalness." In Oxford Studies in Metaphysics, Vol. 8, edited by Karen Bennett and Dean W. Zimmerman, 3-77. Oxford: Oxford University Press.

French, Steven, and Décio Krause. 2006. Identity in Physics: A Historical, Philosophical, and Formal Analysis. Oxford: Oxford University Press.

Halpin, John F. 2003. "Scientific Law: A Perspectival Account.” Erkenntnis 58 (2):137-68.

Hicks, Michael Townsen. 2019. "What Everyone Should Say about Symmetries (and How Humeans Get to Say It)." Philosophy of Science 86 (5):1284-94.

Lange, Marc. 2007. "Laws and Meta-Laws of Nature: Conservation Laws and Symmetries." Studies in History and Philosophy of Modern Physics 38 (3):457-81.

Lange, Marc. 2009. Laws and Lawmakers: Science, Metaphysics, and the Laws of Nature. New York: Oxford University Press.

Lange, Marc. 2011. "Meta-Laws of Nature and the Best System Account." Analysis 71 (2):216-22.

Lange, Marc. 2016. Because without Cause: Non-Causal Explanations in Science and Mathematics. New York: Oxford University Press.

Lewis, David. 1979. “Counterfactual Dependence and Time’s Arrow.” Noûs 13 (4):455-76.

Lewis, David. 1983. "New Work for a Theory of Universals." Australasian Journal of Philosophy 61 (4):343-77.

Lewis, David. 1986a. Philosophical Papers, Volume II. Oxford: Oxford University Press.

Lewis, David. 1986b. On the Plurality of Worlds. Oxford: Basil Blackwell.

Loewer, Barry. 1996. "Humean Supervenience." Philosophical Topics 24 (1):101-27.

McKenzie, Kerry. 2014. "In No Categorical Terms: A Sketch for an Alternative Route to a Humean Interpretation of Laws." In New Directions in the Philosophy of Science, edited by Marcia Carla Galavotti, Stephan Hartmann, Marcel Weber, Wenceslao J. Gonzalez, Dennis Dieks, and Thomas Uebel, 45-61. Berlin: Springer.

Post, Heinz R. 1971. "Correspondence, Invariance and Heuristics: In Praise of Conservative Induction." Studies in History and Philosophy of Science 2 (3):213-55.

Roberts, John T. 1999. "'Laws of Nature' as an Indexical Term: A Reinterpretation of the Best-System Analysis." Philosophy of Science 66 (S3):S502-S511.

Roberts, John T. 2008. The Law-Governed Universe. Oxford: Oxford University Press.

Schaffer, Jonathan. 2005. "Quiddistic Knowledge.” Philosophical Studies 123 (1):1-32.

Schrenk, Markus. 2008. "A Theory for Special Science Laws." In Selected Papers Contributed to the Sections of GAP.6, 6th International Congress of the Society for Analytical Philosophy, edited by Helen Bohse, K. Dreimann, and Sven Walter, 121-31. Paderborn: Mentis.

Schrenk, Markus. 2014. "Better Best Systems and the Issue of CP-Laws." Erkenntnis 79 (10):1787-99.

Tappenden, Jamie. 2008. "Mathematical Concepts and Definitions." In The Philosophy of Mathematical Practice, edited by Paolo Mancosu, 256-75. Oxford: Oxford University Press.

van Fraassen, Bas C. 1989. Laws and Symmetry. Oxford: Clarendon Press.

Wigner, Eugene. 1964a. "Events, Laws of Nature, and Invariance Principles." Science 145 (3636):995-99.

Wigner, Eugene. 1964b. "Symmetry and Conservation Laws." Proceedings of the National Academy of Sciences of the United States of America 51 (5):956-65.

Yudell, Zanja. 2013. "Lange's Challenge: Accounting for Meta-Laws." British Journal for the Philosophy of Science 64 (2):347-69.

Cite this article: Duguid, Callum. 2023. "Symmetries as Humean Metalaws." Philosophy of Science 90 (1): 171-187. https://doi.org/10.1017/psa.2021.48 